University of Massachusetts Amherst

ScholarWorks@UMass Amherst

Physics Department Faculty Publication Series

Physics

1983

\title{
ON THE MIXING OF PSEUDOSCALAR MESONS
}

John F. Donoghue

University of Massachusetts Amherst, donoghue@physics.umass.edu

Harald Gomm

University of Massachusetts Amherst

Follow this and additional works at: https://scholarworks.umass.edu/physics_faculty_pubs

Part of the Physics Commons

\section{Recommended Citation}

Donoghue, John F. and Gomm, Harald, "ON THE MIXING OF PSEUDOSCALAR MESONS" (1983). Physics Letters B. 223.

https://doi.org/10.1016/0370-2693(83)90200-9

This Article is brought to you for free and open access by the Physics at ScholarWorks@UMass Amherst. It has been accepted for inclusion in Physics Department Faculty Publication Series by an authorized administrator of ScholarWorks@UMass Amherst. For more information, please contact scholarworks@library.umass.edu. 


\title{
ON THE MIXING OF PSEUDOSCALAR MESONS
}

\author{
John F. DONOGHUE and Harald GOMM \\ Department of Physics and Astronomy, University of Massachusetts, Amherst, MA 01003, USA
}

Received 17 September 1982

We calculate the mixing between a glueball and $q \bar{q}$ pseudoscalars (both the ground state and the first radial excitation) in the framework of the MIT bag model. Our results are large, strongly violate SU(3) and are very sensitive to small changes in the parameters of the model.

In the quark model, the most difficult states to understand are the isoscalar $J^{P C}=0^{-+}$particles, the $\eta$ and $\eta^{\prime}$. Recently more attention has been focused on this system because of the discovery [1] of the $\iota(1440)$ in $\psi$ radiative decays, which may be a glueball [2] with the same quantum numbers, and also the $\eta(1275)$ seen in the $\pi p$ scattering [3], which probably is a radially excited pseudoscalar. Many related aspects of physics are applicable for these particles: chiral symmetry, the $U(1)$ problem, glueballs and mixings. No description yet has succeeded in

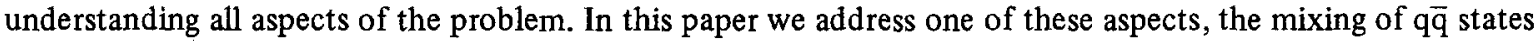
with a glueball. This is of interest for the phenomenology of the pseudoscalar system.

The approach which we use is relatively naive. We use $\mathrm{q} \overrightarrow{\mathrm{q}}$ states constructed in the cavity approximation to the MIT bag model, and consider the lightest $0^{-+}$bag model glueball. The lowest lying $q \vec{q}$ states thus obtained have well known difficulties in describing the $\eta$ and $\eta^{\prime}$ particles (4). One of the implicit assumptions in our approach is then that the physics which alleviates these difficulties does not strongly modify the $\eta$ and $\eta^{\prime}$ wavefunctions. We also consider the first radially excited states. The mixing proceeds through the diagram of fig. 1 , which we calculate by summing over the intermediate states. The question of mixing in this system has also been discussed by Carlson and Hansson [5], and we will comment on their work below. We also discuss the reliability of such a calculation within the bag model context, and find reason for doubting the reliability.

The question of mixing is usually addressed by considering the mass matrix for particles of the same quantum numbers, where the diagonal elements are the bare masses of the states and the off diagonal elements are the mixing matrix elements. It is the latter which we are calculating in the bag model. The basic QQ $\bar{Q} G$ interaction hamiltonian density in QCD is

$H_{\mathrm{I}}(x)=g \bar{\psi}(x) \gamma_{\mu} \frac{1}{2} \lambda^{A} \psi(x) A^{\mu A}(x)$,

and the diagrams of fig. 1 are second order in $H_{\mathrm{I}}$. The effective hamiltonian for GG $\leftrightarrow \mathrm{q} \overline{\mathrm{q}}$ is constructed by standard

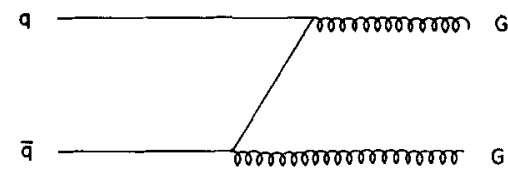

(a)

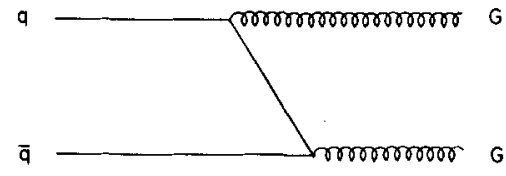

(b)

Fig. 1. The lowest order diagrams for the mixing between a glueball and $q \bar{q}$ pseudoscalars. 
field theory methods

$H_{\text {eff }}=\int \mathrm{d}^{3} x \mathrm{~d}^{4} y T\left(H_{\mathrm{I}}(x) H_{\mathrm{I}}(y)\right)$.

For the intermediate quark line, we construct the bag propagator by inserting a complete set of bagged quarks and summing over the modes.

The lightest pseudoscalar glueball is constructed with the bag model from two gluons ${ }^{\neq 1}$, one in the TE mode $\left(J^{P}=1^{+}\right)$with energy $\omega_{\mathrm{E}}=2.744 / R$ and one in the TM model $\left(J^{P}=1^{-}\right)$with energy $\omega_{\mathrm{M}}=4.493 / R$. Because the gluons are in $l=1$ states and the final state quarks are in $S$ waves, only $J=1 / 2,3 / 2$ intermediate state quarks contribute to the sum over states. At each value of $J$ there are two parities contributing so that there are four types of intermediate states $\mathrm{S}_{1 / 2}, \mathrm{P}_{1 / 2}, \mathrm{P}_{3 / 2}, \mathrm{D}_{3 / 2}$. The explicit quark and gluon wavefunctions are:

$$
\begin{aligned}
& \psi_{\mathrm{S}_{1 / 2}}=\frac{1}{R^{3 / 2}}\left(\begin{array}{c}
\mathrm{i} u_{\mathrm{S}} \chi_{m}^{1 / 2} \\
l_{\mathrm{S}}(\sigma \cdot \hat{r}) \chi_{m}^{1 / 2}
\end{array}\right)=N_{\mathrm{S}}\left(\begin{array}{c}
\mathrm{i}_{\mathrm{o}}\left(x_{\mathrm{S}} r / R\right) \chi_{m}^{1 / 2} \\
-[(E-m) /(E+m)]^{1 / 2} j_{1}\left(x_{\mathrm{S}} r / R\right)(\sigma \cdot \hat{r}) \chi_{m}^{1 / 2}
\end{array}\right), \\
& \psi_{\mathrm{P}_{1 / 2}}=\frac{1}{R^{3 / 2}}\left(\begin{array}{c}
\mathrm{i} l_{\mathrm{P} 1}(\sigma \cdot \hat{r})_{m}^{1 / 2} \\
u_{\mathrm{P} 1} \chi_{m}^{1 / 2}
\end{array}\right)=N_{\mathrm{P} 1}\left(\begin{array}{c}
-\mathrm{i}[(E+m) /(E-m)]^{1 / 2} j_{1}\left(x_{\mathrm{P} 1} r / R\right)(\sigma \cdot \hat{r}) \chi_{m}^{1 / 2} \\
j_{\mathrm{o}}\left(x_{\mathrm{P} 1} r / R\right) \chi_{m}^{1 / 2}
\end{array}\right), \\
& \psi_{\mathrm{P}_{3 / 2}}=\frac{1}{R^{3 / 2}}\left(\begin{array}{c}
\mathrm{i} u_{\mathrm{P} 3} \chi_{m}^{3 / 2} \\
l_{\mathrm{P} 3}(\sigma \cdot \hat{r}) \chi_{m}^{3 / 2}
\end{array}\right)=N_{\mathrm{P} 3}\left(\begin{array}{c}
\mathrm{i} j_{1}\left(x_{\mathrm{P} 3} r / R\right) \chi_{m}^{3 / 2} \\
-[(E-m) /(E+m)]^{1 / 2} j_{2}\left(x_{\mathrm{P} 3} r / R\right)(\sigma \cdot \hat{r}) \chi_{m}^{3 / 2}
\end{array}\right), \\
& \psi_{\mathrm{D}_{3 / 2}}=\frac{1}{R^{3 / 2}}\left(\begin{array}{c}
-\mathrm{i} l_{\mathrm{D}}(\sigma \cdot \hat{r}) \chi_{m}^{3 / 2} \\
u_{\mathrm{D}} \chi_{m}^{3 / 2}
\end{array}\right)=N_{\mathrm{D}}\left(\begin{array}{c}
+\mathrm{i}[(E+m) /(E-m)]^{1 / 2} j_{2}\left(x_{\mathrm{D}} r / R\right)(\boldsymbol{\sigma} \cdot \hat{r}) \chi_{m}^{3 / 2} \\
j_{1}\left(x_{\mathrm{D}} r / R\right) \chi_{m}^{3 / 2}
\end{array}\right), \\
& A_{\mathrm{TE}}=\left(N_{\mathrm{E}} / R\right) j_{1}\left(x_{\mathrm{E}} r / R\right)\left(\varepsilon_{m} \times \hat{r}\right), \quad A_{\mathrm{TM}}=\left(N_{\mathrm{M}} / R\right)\left[j_{2}\left(x_{m} r / R\right)\left(\varepsilon_{m} \cdot \hat{r} \hat{r}-\frac{1}{3} \varepsilon_{m}\right)+\frac{2}{3} j_{\mathrm{o}}\left(x_{m} r / R\right) \varepsilon_{m}\right],
\end{aligned}
$$

where $x_{i}(i=\mathrm{S}, \mathrm{P} 1, \mathrm{P} 3, \mathrm{D})$ are the dimensionless wave numbers for the specific quark modes, determined by

$-\mathrm{i} \hat{\boldsymbol{r}} \cdot \boldsymbol{\gamma} \psi=\psi$

on the surface, $x_{\mathrm{E}(\mathrm{M})}=\omega_{\mathrm{E}(\mathrm{M})} R$, and $\chi_{m}^{1 / 2,3 / 2}$ are the two-component spinors (for $j=l+1 / 2$ ). The quark wavefunctions are normalized such that

$$
\int \mathrm{d}^{3} x \psi^{+}(x) \psi(x)=1
$$

while the gluons are normalized so that the hamiltonian is

$H=\frac{1}{2} \int \mathrm{d}^{3} x\left(E^{2}+B^{2}\right)=\sum_{i} \frac{x_{i}}{R} \frac{1}{2}\left(a_{i} a_{i}^{+}+a_{i}^{+} a_{i}\right)$.

We will use the same bag radius $(R=1 \mathrm{fm})$ for the quark and gluon states, and use $m_{\mathrm{u}}=m_{\mathrm{d}}=0, m_{\mathrm{s}}=300 \mathrm{MeV}$.

It is then relatively straightforward to take the matrix element of the effective hamiltonian. For a single flavor of quark we find

\$1 Contrary to many statements in the literature, there is no problem with the quadratic boundary condition for pseudoscalar (or scalar) glueballs. The boundary condition is satisfied uniformly on the surface, and is equal to the constraint of energy minimization. 


$$
\begin{aligned}
& \left\langle\mathrm{q} \overline{\mathrm{q}}\left|H_{\mathrm{eff}}\right| \mathrm{GG}\right\rangle=\frac{32 \pi \alpha_{\mathrm{S}} N_{\mathrm{E}} N_{\mathrm{M}}}{9 R} \sum_{\text {modes }}\left\{\left[\left(\omega_{\mathrm{S}}+\omega_{\mathrm{E}}-\omega_{0}\right)^{-1}+\left(\omega_{\mathrm{S}}-\omega_{\mathrm{M}}+\omega_{\mathrm{Q}}\right)^{-1}\right] M_{\mathrm{S}}\right. \\
& +\left[\left(\omega_{\mathrm{P} 1}+\omega_{\mathrm{M}}-\omega_{0}\right)^{-1}+\left(\omega_{\mathrm{P} 1}-\omega_{\mathrm{E}}+\omega_{0}\right)^{-1}\right] M_{\mathrm{P} 1}+\left[\left(\omega_{\mathrm{P} 3}+\omega_{\mathrm{M}}-\omega_{0}\right)^{-1}+\left(\omega_{\mathrm{P} 3}-\omega_{\mathrm{E}}+\omega_{0}\right)^{-1}\right] M_{\mathrm{P} 3} \\
& \left.\quad+\left[\left(\omega_{\mathrm{D}}+\omega_{\mathrm{E}}-\omega_{0}\right)^{-1}+\left(\omega_{\mathrm{D}}-\omega_{\mathrm{M}}+\omega_{0}\right)^{-1}\right] M_{\mathrm{D}}\right\},
\end{aligned}
$$

with

$$
\begin{aligned}
& M_{\mathrm{S}}=\int_{0}^{1} y^{2} \mathrm{~d} y\left(u_{\mathrm{o}} l_{\mathrm{S}}+u_{\mathrm{S}} l_{\mathrm{o}}\right) j_{1}^{(\mathrm{E})} \int_{0}^{1} z^{2} \mathrm{~d} z\left[2 j_{\mathrm{o}}^{(\mathrm{M})} u_{\mathrm{o}} u_{\mathrm{S}}+\left(\frac{2}{3} j_{\mathrm{o}}^{(\mathrm{M})}-\frac{4}{3} j_{2}^{(\mathrm{M})}\right) l_{\mathrm{o}} l_{\mathrm{S}}\right], \\
& M_{\mathrm{P} 1}=\int_{0}^{1} y^{2} \mathrm{~d} y\left(u_{\mathrm{o}} l_{\mathrm{P} 1}-u_{\mathrm{P} 1} l_{\mathrm{o}}\right) j_{1}^{(\mathrm{E})} \int z^{2} \mathrm{~d} z\left[2 j_{\mathrm{o}}^{\mathrm{M}} u_{\mathrm{o}} u_{\mathrm{P} 1}-\left(\frac{2}{3} j_{\mathrm{o}}^{(\mathrm{M})}-\frac{4}{3} j_{2}^{(\mathrm{M})}\right) l_{\mathrm{o}} l_{\mathrm{S}}\right], \\
& M_{\mathrm{P} 3}=\int_{0}^{1} y^{2} \mathrm{~d} y\left(u_{\mathrm{o}} u_{\mathrm{P} 3}+l_{\mathrm{o}} l_{\mathrm{P} 3}\right) j_{1}^{(\mathrm{E})} \int z^{2} \mathrm{~d} z\left[j_{2}^{(\mathrm{M})} u_{\mathrm{o}} l_{\mathrm{P} 3}-\left(\frac{4}{3} j_{\mathrm{o}}^{(\mathrm{M})}+j_{2}^{(\mathrm{M})}\right) u_{\mathrm{P} 3} l_{\mathrm{o}}\right], \\
& M_{\mathrm{D}}=\int_{0}^{1} y^{2} \mathrm{~d} y\left(u_{\mathrm{o}} u_{\mathrm{D}}+l_{\mathrm{o}} l_{\mathrm{D}}\right) j_{1}^{(\mathrm{E})} \int z^{2} \mathrm{~d} z\left[j_{2}^{(\mathrm{M})} u_{\mathrm{o}} l_{\mathrm{D}}+\left(\frac{4}{3} j_{\mathrm{o}}^{(\mathrm{M})}+j_{2}^{(\mathrm{M})}\right) u_{\mathrm{D}} l_{\mathrm{o}}\right],
\end{aligned}
$$

where the subscript o refers to the lowest $S_{1 / 2}$ state, i.e. the ground state.

Numerical results are obtained by summing the first ten modes in each channel. The dominant contribution comes from the lowest few modes, and the convergence of the sum is quick. For the ground state configurations, we find

$\left\langle\mathrm{u} \overline{\mathrm{u}}\left|H_{\text {eff }}\right| \mathrm{GG}\right\rangle=38.5 \alpha_{\mathrm{s}} \mathrm{MeV}, \quad\left\langle\mathrm{s} \overline{\mathrm{s}}\left|H_{\mathrm{eff}}\right| \mathrm{GG}\right\rangle=156.5 \alpha_{\mathrm{s}} \mathrm{MeV}$,

while for the radially excited states,

$\left\langle\mathrm{u} \overline{\mathrm{u}}\left|H_{\text {eff }}\right| \mathrm{GG}\right\rangle=35.3 \alpha_{\mathrm{s}} \mathrm{MeV}, \quad\left\langle\mathrm{s} \overline{\mathrm{s}}\left|H_{\text {eff }}\right| \mathrm{GG}\right\rangle=-46.6 \alpha_{\mathrm{s}} \mathrm{MeV}$.

If these results are described in an $\mathrm{SU}(3)$ singlet and octet basis, they correspond to

$\left\langle\eta_{0}\left|H_{\text {eff }}\right| \mathrm{GG}\right\rangle=134.5 \alpha_{\mathrm{s}} \mathrm{MeV}, \quad\left\langle\eta_{8}\left|H_{\text {eff }}\right| \mathrm{GG}\right\rangle=-96.5 \alpha_{\mathrm{s}} \mathrm{MeV}$,

for the ground state, and

$\left\langle\eta_{0}^{*}\left|H_{\text {eff }}\right| \mathrm{GG}\right\rangle=13.9 \alpha_{\mathrm{s}} \mathrm{MeV},\left\langle\eta_{8}^{*}\left|H_{\mathrm{eff}}\right| \mathrm{GG}\right\rangle=67.0 \alpha_{\mathrm{s}} \mathrm{MeV}$,

for the excited state.

One can easily see that the mixing elements are large, especially if we use the phenomenological value of the coupling constant $\left(\alpha_{s}=2.2\right)$ found by considering the spin-spin interaction [4]. (It may be, however, that this is not the appropriate value of $\alpha_{\mathrm{s}}$.) The lowest order mixing amplitudes are then $\left\langle i\left|H_{\text {eff }}\right| j\right\rangle /\left(m_{i}-m_{j}\right)$ which can approach unity for some of the states. In addition, note that flavor symmetry, or $\mathrm{SU}(3)$, is badly broken in the matrix elements.

Carlson and Hansson have also studied $\eta-\eta^{\prime}$ glueball mixing [5], and find that the mixing is large, in qualitative agreement with us. However, the technical differences between their method and ours tends to obscure the fact that we are each calculating different quantities. They calculate the overlap of a q $\bar{q}$ state propagated in from $t=-\infty$ to $t=0$ onto a bare GG state, called $\langle G G \mid q \bar{q}\rangle$. This quantity has only half of the poles in the intermediate state as does our mixing amplitude. The other half would appear in the opposite quantity $\langle q \bar{q} \mid G G\rangle$ in which the GG state is propagated in and projected onto a bare $q \bar{q}$ state. In their framework $|\langle G G \mid q \bar{q}\rangle|$ and $|\langle q \bar{q} \mid G G\rangle|$ are not 
equal. While their calculation is a measure of mixing, we feel that it is not what one would normally use in phenomenological applications of mixing. We have attempted to use the conventional mixing methods.

In trying to understand the reason for the large amount of SU(3) breaking, we uncovered a feature which makes us question the reliability of the numbers. The overlap integrals vary smoothly with the quark mass. However, the energy denominators can provide strong dependence on minor changes of the parameters in the model. For example, in the contribution of the $S$ waves, we find the denominator $\omega_{S}-\omega_{M}+\omega_{0}$. For massless quarks in the ground state $\omega_{\mathrm{S}}=\omega_{0}=2.04$ and $\omega_{\mathrm{M}}=4.49 / R$, so that $\omega_{\mathrm{S}}-\omega_{\mathrm{M}}+\omega_{0}=-0.41 / R$. However, for strange quarks with mass $m=300 \mathrm{MeV}, \omega_{\mathrm{S}}=\omega_{0}=2.92 / R$, so that $\omega_{\mathrm{S}}-\omega_{\mathrm{M}}+\omega_{0}=+1.35 / R$. The denominator has changed sign. For $m=80 \mathrm{MeV}$ the denominator vanishes, and the mixing amplitude becomes infinite. Likewise, small higher order corrections to the hadronic energies will shift the denominator closer to or further from the pole, producing large changes in the mixing amplitudes. To the extent that this is a valid physical effect, we should not be surprised by large SU(3) breakings, but the specific numbers should be treated with caution.

An additional feature which affects the reliability of the result concerns our use of equal radii for the $\eta, \eta^{\prime}$ and glueball. Without this assumption the problem is not as well defined, as the procedure for calculating wavefunction overlap of unequal bags is not clear. However, in reality one does expect different radii for the $\eta$ and a glueball. For massless quarks and gluons, the bag virial theorem predicts $R \propto m^{1 / 3}$ where $m$ is the mass of the bound state.

We have studied this effect by using different radii in the wavefunction and cutting the integration off at the smaller radius. The results can depend significantly on this aspect. Again it is primarily the energy denominators which are most important. By changing the radius one changes some of the energy factors in the denominator, producing large changes in the result similar to those discussed above.

To summarize, we have studied the cavity approximation to the MIT bag model and extracted the predictions for the mixings of $\mathrm{q} \overline{\mathrm{q}}$ states with a $\mathrm{O}^{-+}$glueball. The results given correspond to large mixings. However, the values of the mixing amplitude are very sensitive to shifts in quark masses and other parameters, producing large SU(3) breakings and an uncomfortable sensitivity to details of the model.

We thank C. Carlson and H. Hansson for useful communications. This work was supported in part by the National Science Foundation.

\section{References}

[1] C. Edwards et al., Phys. Rev. Lett. 49 (1982) 259.

[2] M. Chanowitz, Phys. Rev. Lett. 46 (1981) 981;

K. Ishikawa, Phys. Rev. Lett. 46 (1981) 978,

J.F. Donoghue and H. Gomm, Phys. Lett. 112B (1982) 409.

[3] N. Stanton et al., Phys. Rev. Lett. 42 (1979) 346.

[4] T. DeGrand, R.L. Jaffe, K. Johnson and J. Kiskis, Phys. Rev. D12 (1975) 2060.

[5] C. Carlson and T.H. Hansson, Nucl. Phys. B199 (1982) 441. 